\title{
Testing of Surface Mounted Superconducting Stacks as Trapped-Flux Magnets in a Synchronous Machine
}

\author{
Vicente Climente-Alarcon, Member, IEEE, Anis Smara, Lukasz Tomkow, Bartek A. Glowacki, \\ Thomas Reis
}

\begin{abstract}
Stacks of high temperature superconducting tapes may offer a technically affordable solution for the application of superconducting materials as trapped-flux magnets in the rotor of synchronous electrical machines. Nevertheless, several concerns must be first addressed, among others, the optimal procedure to induce the current vortexes previously to operation (magnetization), the survivability of these vortexes in the electromagnetic environment present in an electrical machine and the accuracy of recently developed numerical models. With the aim of exploring such practicalities, this paper presents a modified synchronous machine to test under liquid nitrogen conditions thin stacks of superconducting tapes. The machine is run under realistic conditions: currents in the stacks are induced from the stator, then the shaft is rotated and finally the machine is connected to a load, working as a generator. The results confirm previous numerical and experimental studies and establish a procedure for assessing the behavior of stacks in their actual operational environment.
\end{abstract}

Index Terms - AC Motors, high temperature superconductors, numerical simulation, superconducting magnets, demagnetization.

\section{INTRODUCTION}

$\mathrm{A}$ $\mathrm{n}$ increase in the power density of rotating electrical machines would open new fields of application where lightweight is paramount, such as aircraft propulsion [1] and have an important impact in others such as wind generation [2]. In synchronous machines an initial approach for achieving this objective would consist of improving the value of the magnetic flux density provided by the rotor by means of superconducting technology [3].

In this line, trapped-flux magnets composed of stacked layers of high temperature superconducting (HTS) tape offer in principle a simpler solution compared to other choices. First, they hold the actual trapped flux record at $17.7 \mathrm{~T}$ [4], achieved thanks to their good mechanical characteristics, inherited from the substrate, usually a superalloy, on which the

This manuscript was submitted for review on November 26, 2019.

This research is financially supported by the European Union's Horizon 2020 research innovation programme under grant agreement No 723119 (ASuMED consortium) and EPSRC grant EP/P000738/1.

Vicente Climente-Alarcon, Anis Smara, Lukasz Tomkow and Bartek A. Glowacki are with the Applied Superconductivity and Cryoscience Group, superconducting layer is deposited. In addition, bending, to follow the rotor surface [5] or to achieve optimal shapes from the electromagnetic point of view is possible [6]. This substrate is also responsible for better thermal conductivity, which prevents flux jumps as it happens in bulks for temperatures below 20-30 K [7].

Stacks also portray improved manufacturability and reliability compared to bulks or coils, since they are formed just by pilling up layers of tape joined either by a plastic binder or by soldering [8]. In this way, any point defect is smoothed out by the added contribution of all the layers. For the same reason, quench in stacks is a slower, easily detectable, phenomenon [9].

Finally, compared to coils used as field windings [2], trapped-flux magnets such as stacks provide a simpler machine construction with no need of current leads. Thus, they act as stand-alone permanent magnets. This reduces the complexity of the rotor, a machine's subassembly that must already be built to endure important mechanical loads and thermal gradients; and feature the cooling systems necessary to maintain the superconductor at cryogenic temperatures [10].

The methods for designing trapped-flux magnets are well established. Their electromagnetic behavior can be reproduced using Finite Element (FE) analysis by means of the $H$ formulation, as it was done in [4] to study field cooling magnetization. This approach can be extended to stacks as long as they are treated as homogenized regions. However, the $H$ formulation is more resource exhaustive than the usual $A$ formulation utilized in the design of conventional electrical machines. This contrast that separated the superconductor's and electrical machine's fields was solved by a more recent methodology that weakly couples both the $H$ - and $A$ formulations on commercial software. The technique provides a straightforward way of introducing superconducting regions in the established computational design procedure of electrical motors, combining both fields from the numerical point of view [11]. This computational approach has been extensively utilized during the design of the first fully superconductive motor demonstrator for evaluating different rotor architectures and

University of Cambridge, CB3 0FS, Cambridge, UK, (e-mails: vc363@cam.ac.uk, as2903@cam.ac.uk,1tt27@cam.ac.uk,bag10@cam.ac.uk).

Bartek A. Glowacki is also with The Institute of Power Engineering, ul. Mory 8, 01-330 Warsaw, Poland.

Thomas Reis is with Oswald Elektromotoren GmbH, 63897 Miltenberg, Germany (email: thomas.reis@oswald.de). 
field cooling magnetization procedures [6, 12], although an experimental evaluation of its accuracy was pending.

In addition to this design-related question, several others related to their operation inside the machine arise. On one hand the current vortexes circulating in the stacks must be induced before the motor can yield torque. In laboratory conditions this is achieved by either field cooling [7] or zero field cooling procedures, with pulse magnetization included in the later [8]. On the other hand, those supercurrents must be left unperturbed during operation. This is not an easy task since any variation of the magnetic flux density will cause losses, if it is normal to the direction of magnetization [13], and demagnetization, if it is orthogonal (cross-field) [14] and these variations are always present in the airgap of an electrical machine in the form of spatial harmonics $[15,16]$.

Experimental results suggest that the rate of demagnetization of stacks is lower, at least initially, than in bulks [17] due to the high aspect ratio of the stack's layers. Moreover, such effect is damped at lower operating temperatures [18], since the value of the penetration field increases with the critical current $J_{c}$. Nonetheless, it remains still unclear if the actual conditions of operation inside of an electrical machine affect them and in what degree the widening results obtained in laboratory by the academic community about the behavior of stacks under crossfields [18-20], and even rotating fields [20], can be translated into the actual electromagnetic environment existing inside a motor.

Regarding these questions, initial experiments were carried out in [21], in which only one stack was installed on the rotor of an otherwise conventional motor and submerged in a liquid nitrogen bath. The stack was pulse magnetized using one of the existing coils in the stator and spun. Other coils supplied cross fields that allowed the evaluation of the demagnetization rate profile for thousands of cycles. However, the demagnetization rate in realistic (energy conversion) operational conditions was not tested then.

This work addresses by performing an experiment both of the above mentioned questions: the evaluation of the accuracy of novel numerical methods devised to designing rotating electrical machines with superconducting regions $[6,12]$ and the assessment of the demagnetization rate under actual operational conditions of these machines. In this experiment the machine used in [21] has been configured as a 4-pole pair generator setting 8 stacks around the surface of its rotor and its stator winding has been connected to a load through a circuit breaker. Currents in the stacks are induced by pulse magnetization with the machine at standstill, then the rotor is spun, and the electromotive force induced by the stacks at open circuit in the terminals of the machine is measured. This magnitude allows a direct comparison with the output of numerical models, as it is done in conventional machines. Finally, the circuit breaker is closed and currents flow in the generator, where the demagnetization rate caused by the stator winding's circulating currents, that is, under realistic energy conversion conditions, is evaluated.

Therefore, the outcomes of this work are:

1) To show an approach for testing superconducting trapped- flux magnets under realistic conditions.

2) To provide an insight into the accuracy of the numerical methodology proposed in [11] and used in $[6,12]$ to assess different rotor configurations of a fully superconducting motor.

3) To evaluate the demagnetization rate of surface mounted stacks in the actual electromagnetic conditions of an electrical machine.

With this aim the remainder of the paper is outlined as follows: Section II describes the characteristics the of the commercial machine used, the modifications carried out to fix and magnetize the stacks and the calculations to select the load. Section III provides an overview of the FE method used and the specific modifications performed for this work, Section IV presents the results, and finally, Section $\mathrm{V}$ yields the conclusions.

\section{EXPERIMENTAL ARRANGEMENT}

The steps taken to configure an otherwise conventional machine to work as a generator featuring stacks as source of magnetic flux density in the rotor are explained in this section.

\section{A. Machine's characteristics}

The generation experiment has been carried out in the same machine used in [21], that is, a slightly modified conventional one supplied by Oswald Elektromotoren $\mathrm{GmbH}$, having the characteristics shown in Table I. The stator winding was manufactured in a double-layer fractional slot non-overlapping configuration featuring 12 slots, whereas the rotor is a cylindrical laminated one.

The fractional slot stator configuration provides the flexibility to arrange a greater number of pole pairs [16], however, only eight stacks are fitted around the perimeter of the rotor, which conforms a 4 pole-pair machine. This was done to facilitate the magnetizing process from the stator.

The existing stator winding (copper coils in Fig. 1 b) ) is connected by joining the ends of the coils in a 3 phase $/ 4$ pole pair configuration (A, B, C coils in Fig. 1 a) ). These coils so arranged act as an armature winding, that is a winding immersed in a rotating field (created by the stacks) where the voltage required for energy conversion is induced.

TABLE I

GEOMETRIC AND ELECTRIC CHARACTERISTICS OF THE MACHINE

\begin{tabular}{lc} 
Stator external diameter & $236 \mathrm{~mm}$ \\
Stator internal diameter & $120 \mathrm{~mm}$ \\
Airgap length & $2 \mathrm{~mm}$ \\
Stack width & $41 \mathrm{~mm}$ \\
Airgap length & $2 \mathrm{~mm}$ \\
Stack thickness & $1 \mathrm{~mm}$ \\
G10 sleeve thickness & $1 \mathrm{~mm}$ \\
Rotor yoke external diameter & $114 \mathrm{~mm}$ \\
Rotor yoke internal diameter & $36 \mathrm{~mm}$ \\
Machine length & $160 \mathrm{~mm}$ \\
Number of pole pairs & 4 \\
Number of phases & 3 \\
Armature coil turns & 22 \\
Number of stator slots & 12 \\
Magnetizing coil turns & 8 \\
Peak magnetizing current & $400 \mathrm{~A}$ \\
\hline \hline
\end{tabular}




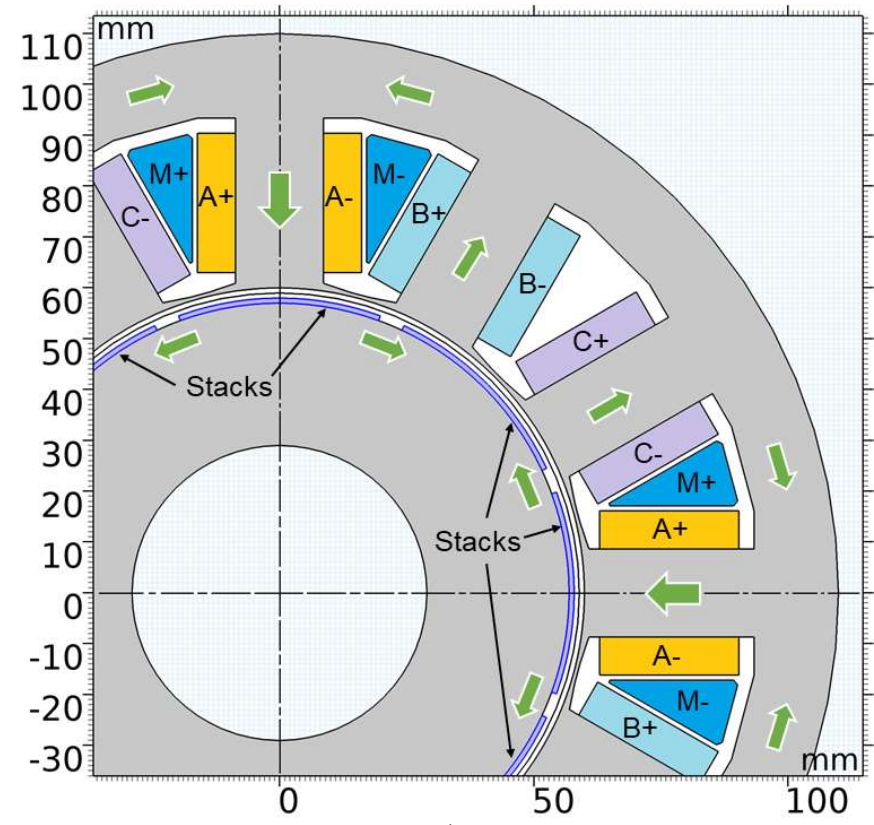

a)

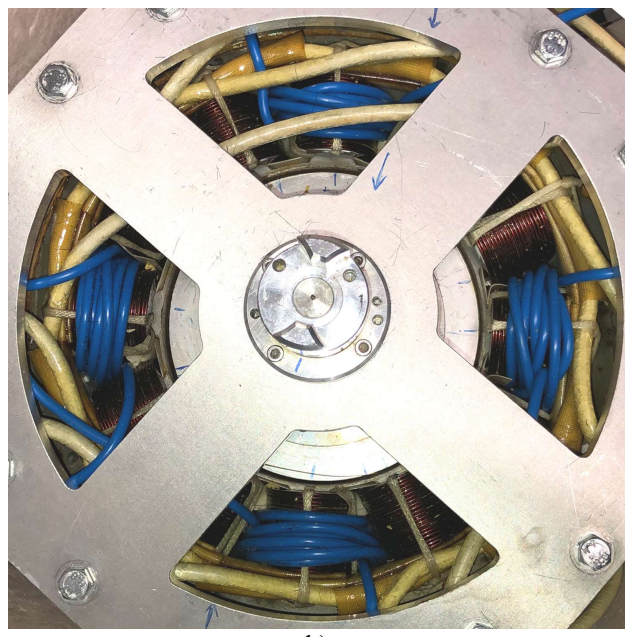

b)

Fig. 1. Simulated geometry displaying the cross-section of the machine. The superconducting stacks embedded in the rotor yoke are depicted in blue, a) Assembled machine showing the magnetizing coils in blue over the motoring winding below the top flange and coupling, $b$ ).

In this experiment, 8 stacks each built up using 9 layers of AMSC (RE)BCO tape [4] are cut to a width of $41 \mathrm{~mm}$ and fixed on the rotor's surface by Kapton tape and the slight compression of a G10 sleeve (Fig. 2). This tape features a critical current of $391 \mathrm{~A} / \mathrm{cm}$-width at $77 \mathrm{~K}$ and self-field, and an engineering current of $4.49 \cdot 10^{8} \mathrm{~A} / \mathrm{m}^{2}$ [4]. According to the measurements made in a Hall scan probe, the estimated flux density that each layer of tape can trap is $22 \mathrm{mT}$ at $77 \mathrm{~K}$. This provides a theoretical peak of $0.2 \mathrm{~T}$ on each stack.

During the experiment the generator so configured is introduced in an insulated bucket, filled with liquid nitrogen as seen in Fig. 1, hence all thermal effects on the stacks are damped due to the rapid evaporation of the surrounding coolant. This condition departs from the actual ones during the operation of a superconducting rotating electrical machine; therefore, the effect of losses cannot be evaluated by means of this setup and remains a subject of research.

The generator's input torque is provided by a speedcontrolled DC motor coupled to the shaft above the surface level of the liquid nitrogen (not shown in Fig. 1 b) ). It allows changing the generator's turning rate in the range of 10 to 35 $\mathrm{Hz}$.

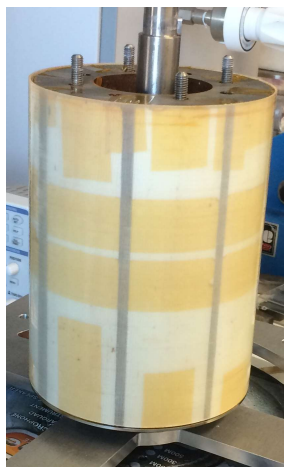

Fig. 2. Rotor used during the experiment featuring 8 AMSC stacks underneath a G10 sleeve. The stack themselves are supported by Kapton tape and positioned relative to each other by the same means.

\section{B. Magnetizing circuit}

As in [21] pulse magnetization was chosen to induce the currents in the stacks because it is a simpler procedure and the availability of a capacitor bank in the laboratory. However, contrary to [21] the motoring winding is supplemented by a manually wound one specifically dedicated to magnetization. This modification was considered to avoid stressing the original coils with further pulsing. The new magnetization winding is installed in the clearance left between the original copper coils and wound around each tooth ( $\mathrm{M}$ winding in Fig. 1 a), blue cables in Fig. 1 b) ). It features just one coil group, seriesconnected, with 8 turns around every third teeth, yielding a symmetric configuration with four poles circulating flux towards the rotor spaced 90 degrees each. Outward facing poles cannot be wound since there is no tooth directly in front of the corresponding stacks (see Fig. 1 a) ). The ends of this magnetization winding are connected to a capacitor bank [8]. In this way, during pulse magnetization, flux is circulated against 4 of the 8 stacks. It was hypothesized that the remaining would be magnetized by the outward-bound circulation of this same flux. Green arrows in Fig. 1 a) depict the magnetic flux density circulation average path during pulsing.

Furthermore, it must be observed that although the new winding features a low voltage insulation effectively withstanding between 600 and $1000 \mathrm{~V}$, the full pulsing power of the capacitor bank cannot be used, since due to symmetries of the machine, the magnetizing winding shares the same teeth as one phase of the armature winding (A phase in Fig. 1). Therefore, the voltage induced in that phase is increased by the turns ratio in each tooth, that is $22 / 8$, tripling the one supplied by the capacitors, which can damage its insulation. That is, the magnetizing winding and the phase $\mathrm{A}$ of the armature winding are coupled in a similar way of a the primary and secondary circuits of a transformer. 


\section{Load design}

Regarding the motoring circuit, the armature winding (A, B, $\mathrm{C}$ in Fig. 1 a) ) is connected in star configuration (see Fig. 3), as usually done in electrical machines to avoid the circulation of the third harmonic. Every third coil in the machine is seriesconnected to the same phase, forming a group. One end of each group is grounded at the center of the star, whereas the other is led out of the bucket into a circuit breaker and the load. When the circuit breaker is open, measurements of the induced electromotive force in one phase of the generator can be performed by a voltmeter (see Fig. 3) without the influence of the load or the voltage drop contributed by the internal reactance of the machine. When the circuit breaker is closed, currents will circulate. These currents will modify the magnetic flux density distribution in the airgap of the machine (effect known as armature reaction) and their influence on the stacks can be assessed. The value of this currents will depend on the load connected, whose resistance value is estimated in this subsection.

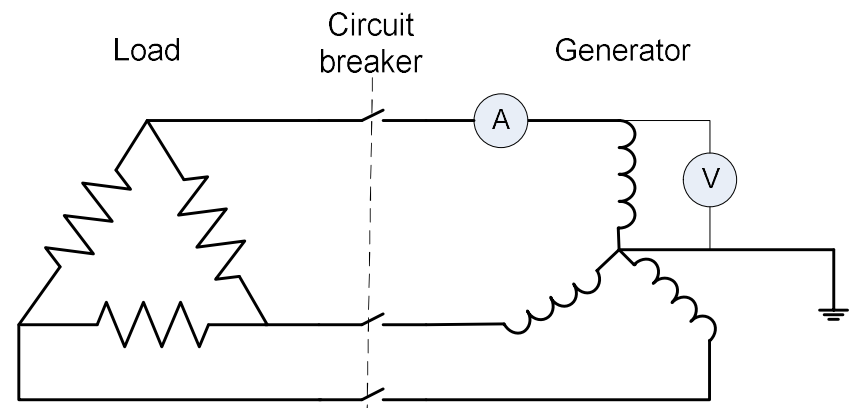

Fig. 3. Connections between the load (left hand side) and the generator's armature winding (right hand side) during the experiment. The magnetizing winding is not depicted.

For this load estimation, first it must be considered that although the experiment is aimed at operating the setup as a generator, the actual values of the electromagnetic quantities inside a typical electrical machine cannot be reproduced. The clearance left between rotor and stator and the cost of wide superconductor tape, as well as the difficulties in magnetization, prevent obtaining above $1 \mathrm{~T}$ of magnetic flux density in the airgap, a usual value in permanent magnet synchronous motors. This limitation would yield lower voltage and therefore smaller produced current.

Since the circuit in Fig. 3 is symmetric, it is analyzed considering per phase basic electric circuit composed of an alternating voltage source, an inductance and a resistor in series:

$$
E=R_{\text {star }} \cdot i_{\text {phase }}+L_{\text {stator }}\left(i_{\text {phase }}\right) \cdot \frac{d i_{\text {phase }}}{d t}
$$

where $R_{\text {star }}$ is the resistance of the load, converted from delta to star, $L_{\text {stator }}$ the inductance of the generator and $i_{\text {phase }}$ the current circulating in that branch of the circuit. The available electromotive force $E$ yielded by the machine is approximated by a well-known expression used in electrical engineering, adapted from Faraday's law:

$$
E=\frac{2 \pi}{\sqrt{2}} K \cdot f \cdot N \cdot \widehat{\phi_{m}}
$$

where $K$ is the winding factor, $f$ the electrical frequency, related to the rotational frequency by dividing it by the number of pole pairs, $N$ the total number of turns in one phase and $\widehat{\phi_{m}}$ the peak flux amplitude in one coil assumed it as a sinusoidal variation in the airgap. For a spinning frequency of $11 \mathrm{~Hz}$ and $100 \mathrm{mT}$ of maximum flux, the same conditions as in [21], the available electromotive force is $4.46 \mathrm{~V}_{\mathrm{rms}}$. The winding factor is taken from [16].

Furthermore, inside the machine there are other effects that limit the circulation of current. The main contribution comes from the stator inductance itself $L_{\text {stator }}$ (also known as synchronous reactance), which is difficult to estimate by analytical means, due to the presence of several paths in the magnetic circuit. Moreover, since this stator feature teeth bridges that close each slot, it is expected that $L_{\text {stator }}=$ $L_{\text {stator }}\left(i_{\text {phase }}\right)$, that is, the stator inductance changes sharply with the current fed into the stator as the bridges saturate, yielding a very non-linear variation of $L_{\text {stator }}$ at low currents. Therefore the value of its inductance was computed by conventional FE analysis [22]. The results per phase are shown in Fig. 4, confirming this non-linearity.

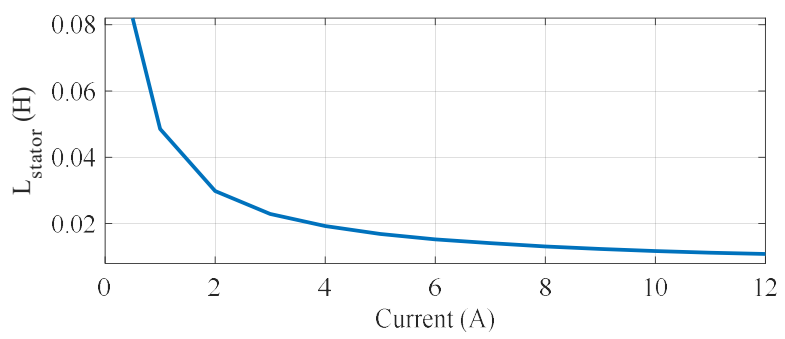

Fig. 4. Variation of stator inductance with armature current.

This result poses a problem: according to [21] currents of several amperes in the stator are needed to obtain the experimental resolution necessary to measure the demagnetization rate of the stacks before the liquid nitrogen supply runs out. Thus, the high value of the inductance $L_{\text {stator }}$ shown in Fig. 4 for low currents and the relatively low value of the electromotive force induced in the generator coils $E$ prompted, according to (1), to the selection of a load $R_{\text {star }}$ as small as technically possible. For these reasons the load used in this experiment is delta-connected and composed of a bank of resistors with $410 \mathrm{~m} \Omega$ each. This maximizes the current circulating in each phase.

\section{FINITE ELEMENT MODEL}

The electromagnetic state of the generator's cross section shown in Fig. 1 a) is simulated using the weakly coupled approach proposed by Grilli et al. in [11] implemented in the commercial software COMSOL. In the stacks and surrounding 
regions, the value of the magnetic field $\boldsymbol{H}$ is solved using (3), being $\mu$ the magnetic permeability and $\rho$ the resistivity of the material [23]:

$$
\frac{\partial \mu \boldsymbol{H}}{\partial t}+\nabla \times \rho(\nabla \times \boldsymbol{H})=0 .
$$

Eq. (3) permits the direct use of the $E-J$ law for the superconducting regions in the form:

$$
\rho=\frac{E_{c}}{J_{c}}\left(\frac{|J|}{J_{c}}\right)^{n-1} .
$$

In this work $J_{c}=J_{c}(|\boldsymbol{B}|, T)$ and $n=n(|\boldsymbol{B}|)$, therefore, no variation of the critical current value with the angle of incidence of the magnetic flux density $\boldsymbol{B}$ is taken into account. The other inputs to (4) are the temperature $T$ and the current density $\boldsymbol{J}=\left[J_{z}\right]$ obtained from solving (3).

The stacks have been modelled as a homogeneous region, that is, behaving as an isotropic bulk with the critical current correspondingly scaled down. Their relative magnetic permeability is taken as constant and thickness proportional to the value extrapolated from manufacturer's data for the Ni5 at $\% \mathrm{~W}$ substrate of the AMSC tape, $\mu_{r}=22$ [24].

The rest of the machine, mainly consisting of the stator regions, is computed by means of the commonly utilized $A$ formulation [22]. Both formulations are weakly coupled at a tramodel boundary established in the airgap, $1 \mathrm{~mm}$ inside the rotor mesh.

By this procedure, a rotating electrical machine having superconductive regions can be simulated as a conventional one, that is, yielding the same magnitudes that are commonly used in the design of an electrical machine, and allowing the same techniques commonly applied to them, such as independent stator and rotor meshes coupled at the airgap [22]. This permits relative movement from each other and thus the turn of the rotor can be reproduced. More information about this approach can be found in $[6,12]$ where it was utilized to model field cooling magnetization and motoring, however, the results in those works came only from simulations, thus, a quantification of this method's accuracy was pending.

In order to simulate the pulse magnetization available in the laboratory, the model used in $[6,12]$ for field cooling is slightly modified (Fig. 5). In this work, at the beginning of the computation the rotor and hence the stacks are already at $77 \mathrm{~K}$. The machine geometry and the experimentally measured pulse current are inputs to the model. The current density in the M winding (see Fig. 1 a) ) is varied accordingly to the values recorded during the experiment of the current circulating in the magnetizing winding. This process lasts in the model $15 \mathrm{~ms}$ after which the simulation yields an estimation of the electromagnetic state of the cross-section of the machine, including the currents in the stacks. To replicate the experimental procedure, a relaxation period of 20 seconds is computed before rotation begins (see Fig. 5). The machine is also at standstill during that period, and no currents are fed to any of the windings. After relaxation the rotor mesh begins to turn and the variation of the magnetic flux density produced in the stator by the moving stacks induces a voltage on the phases $(\mathrm{A}, \mathrm{B}, \mathrm{C})$ of its armature winding. This voltage can be directly compared to the experimental one obtained from the voltmeter in the setup when the circuit breaker in Fig. 3 is open and thus it provides a quantification on the accuracy of the model. This comparison between the numerically computed emf and the experimental one constitutes the most important step for validating a model in conventional machines. All simulation stages are depicted in Fig. 5.

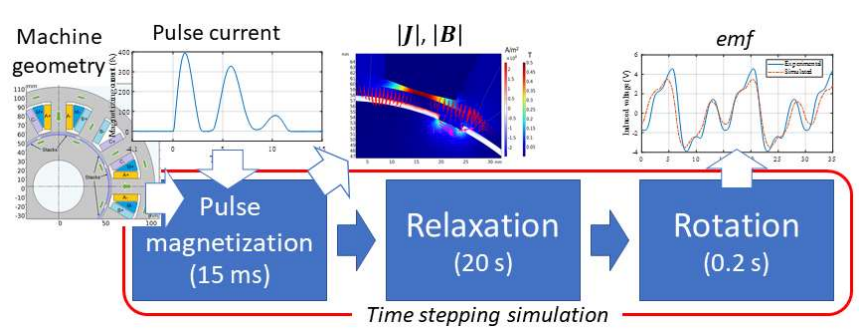

Fig. 5. Simulation procedure followed consisting of three stages: magnetization, relaxation and rotation. The recorded magnetizing current waveform is an input to the model. The outputs are the state of the stacks after magnetization and the electromotive force induced in the stator winding during rotation. This later allows a comparison with experimental measurements.

In addition, the temperature raise during magnetization is also computed. A simplified approach is used for this purpose considering adiabatic conditions in the stacks. Variable heat capacities of the superconductor, the silver layer and the substrate in a wide temperature range around the initial value of $77 \mathrm{~K}$ are considered in the thermal model.

\section{RESUlTS}

The experimental part follows the three stages carried out during the simulation in Fig. 5, plus a fourth one. That is, the stacks are magnetized, a short relaxation period follows and then the DC motor spins the generator's rotor with the circuit breaker in Fig. 3 open. These results are examined in the first point of this section to assess the accuracy of the model. In the fourth stage the flow of currents in the stator armature is allowed by closing the circuit breaker and the demagnetization rate under realistic conditions is checked. These other results are presented in the second part of this section.

\section{A. Numerical computation method assessment}

The initial pulse magnetization procedure consists of manually positioning the rotor with the centerline of four stacks facing the magnetizing coils and supplying just one pulse. More complicated pulsing patterns, such as iteratively magnetizing pulsed field method with reduced amplitude (IMRA), were also tried. However, they were not followed in this test since it was observed experimentally that their output wasn't predictable due to the errors arising from the necessity of repositioning the rotor after some of the pulses. 
During magnetization, a Fluke i1000s AC current clamp was attached to one of the cables supplying the $\mathrm{M}$ coil, configured in the $1 \mathrm{mV} / \mathrm{A}$ range and the magnetizing current waveform recorded. The result of the unique $150 \mathrm{~V}$ pulse employed in this test is shown in Fig. 6 comprising three lobes, lasting $12 \mathrm{~ms}$ and peaking at almost $400 \mathrm{~A}$. This waveform is an input to the numerical model, as illustrated by Fig. 5 .

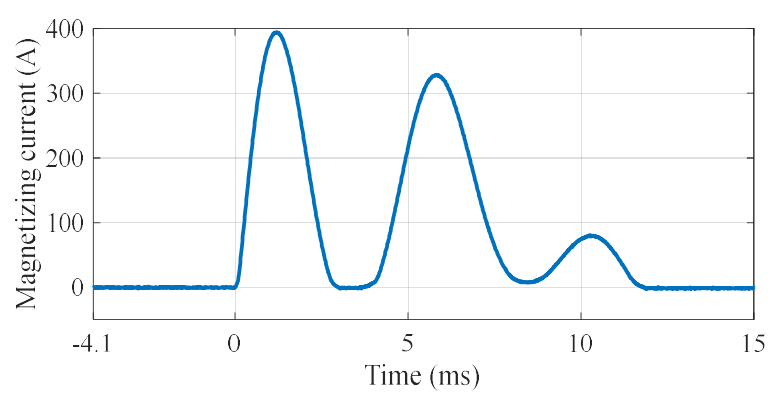

Fig. 6. Current profile in the $M$ (magnetizing) winding during a pulse for the capacitor bank charged to $150 \mathrm{~V}$. The evolution corresponds to an underdamped RLC circuit showing three peaks with the returning current limited by the bank's diodes.

For illustrative purposes, the output of the first stage of the numerical model is shown in Fig. 7 a), displaying the computed electromagnetic state of the cross-section of the motor after magnetization. The different current density distribution in the contiguous stacks is obvious in the picture, which highlights the importance of a correct magnetic circuit design, as already observed in [12]. The fact that the inward flux stacks are facing the center of one tooth whereas the outward ones lay in-between two of them creates an asymmetric magnetic path (green arrows in Fig. 1 a) ) and thus yields different magnetization levels and current distribution patterns in the superconductor. This asymmetry that increases the harmonics in the induced emf can be avoided using a distributed winding, as in $[6,25]$, instead of a tooth-coil one, at the cost of a higher complexity arranging the coils.

The currents circulating in the stacks, calculated as the half value of the integral of $\left|J_{z}\right|$, the current density in the out-ofthe-plane direction, are 1,708 $\mathrm{A}$ in the left-hand side stack, below the magnetizing coil, and $268 \mathrm{~A}$ in the neighboring one. According to the simulations, the trapped flux reaches a peak of $86 \mathrm{mT}$ in the former stack, with an average of $55 \mathrm{mT}$. The later exhibits a peak of $100 \mathrm{mT}$, whereas the average value is below $20 \mathrm{mT}$. In Fig. 7 a), the magnetic flux density around the stacks is represented by arrows in different scales for the airgap (red, $\mathrm{x} 4$ ) and in the rotor iron (magenta, $\mathrm{x} 1$ ).

The coupled thermal finite element simulation, considering adiabatic conditions, reveals a maximum temperature reached during the magnetization process of $79.1 \mathrm{~K}$ at the corner of the inward flux facing stack. Therefore, thermal effects during the magnetization process are negligible.

After the pulse is delivered, the motor is rotated at open circuit conditions (with the circuit breaker in Fig. 3 open) and the emf induced by the stacks in phase A acquired by means of a Yokogawa DL-750 recorder. A sampling rate of 10

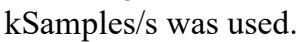

Fig. 7 b) compares the induced back-emf waveform between the simulated and the experimental case during the operation of the machine. For the simulated case, the machine is rotated at $16 \mathrm{~Hz}$, whereas the experimental one turns at $16.33 \mathrm{~Hz}$. This is translated into an electrical frequency of $64 \mathrm{~Hz}$, close to the industrial standard. A good agreement in the waveform's shape is appreciated, where all the components of the signal are correctly reproduced. However, regarding the amplitude the model underestimates the currents in the stacks by $24 \%: 4.57 \mathrm{~V}$ peak are obtained experimentally, whereas the simulated waveform peaks at $3.48 \mathrm{~V}$.

This result confirms the validity of the numerical approach introduced in [11] to reproduce the behavior of an electrical machine with superconducting regions in its rotor, and thus its utility for designing purposes.


b)

Fig. 7. Top, a), simulation showing the effects of the magnetization process. Red-blue scale, current density in the stacks; color scale, magnetic flux density elsewhere. Arrows, circulation of the magnetic flux density. Bottom, b), comparison of the emf waveforms obtained.

This difference of $24 \%$ between the peak values of the biggest component in Fig. 7 a) could be attributed to several causes:

1) The magnetic permeability of the substrate during pulsing varies appreciably, whereas here a constant one has been used. Longer simulations with a variable magnetic permeability have been carried out, with no improvement in the results.

2) Leakage paths are not adequately modelled. Since only the cross section of the machine is studied in the finite element model, 3D effects such as axial leakage, which might be important during pulsing, cannot be assessed. 
TABLE II

DEMAGNETIZATION RATES

\begin{tabular}{cccccc}
\hline \hline Stator current $(A)$ & Winding & $\begin{array}{c}\Delta B \text { Cross-field } \\
(\mathrm{mT})\end{array}$ & $\begin{array}{c}\text { Demagnetization rate } \\
\text { (observed) }\end{array}$ & $\begin{array}{c}\text { Demagnetization rate } \\
\text { (corrected) }\end{array}$ & $\begin{array}{c}\text { Relative } \\
\text { decreasing rate }\end{array}$ \\
\hline 0.6 & Armature, 3 phase & $45-6$ & $-2.2 \cdot 10^{-5} \mathrm{~A} / \mathrm{s}$ & $-5.5 \cdot 10^{-6} \mathrm{~A} / \mathrm{s}$ & $-8.59 \cdot 10^{-6} \mathrm{~s}^{-1}$ \\
3.5 & Two opposite coils & 8 & $-2.3 \cdot 10^{-4} \mathrm{~V} / \mathrm{s} *$ & $-3.9 \cdot 10^{-5} \mathrm{~V} / \mathrm{s}$ & $-3.9 \cdot 10^{-5} \mathrm{~s}^{-1}$ \\
3.5 & Two contiguous coils & 12 & $-1.5 \cdot 10^{-4} \mathrm{~V} / \mathrm{s} *$ & $-2.6 \cdot 10^{-5} \mathrm{~V} / \mathrm{s}$ & $-2.6 \cdot 10^{-5} \mathrm{~s}^{-1}$ \\
\hline \hline
\end{tabular}

* Taken from [21].

Yet, in general this would be detrimental to the magnetization of the stacks and here the contrary outcome is observed.

3) Finally, as said in the previous section, the superconductor's characteristics used in the model were parametrized not considering the dependence of $J_{c}$ with the angle of the magnetic flux density on the surface of the material $\theta$. However, in this machine the magnetic flux density during the magnetization process is mostly tangential to the surface of the stack under the tooth. This is due to the presence of iron bridges between teeth. This effect in general increases $J_{c}$, which may correspond to a higher amplitude of the main peak as observed in the experimental waveform.

Despite this, the results of the experiment and the model agree quite well, especially taking into account the electromagnetically demanding conditions under which the magnetization process is carried out. Hence, these results, although obtained in a laboratory facility instead of industrial conditions, constitute an experimental validation of the finite element model introduced in [11] and presented in Section III. They allow the establishment of an error boundary in the output of previous works aimed at designing a fully superconducting motor $[6,12]$. It is expected for the cases shown in $[6,12]$ that the difference between the calculated and the experimental values be lower than the observed in this experiment, since field cooling magnetization, a less severe electromagnetic transient, will be utilized in the fully superconducting machine.

\section{B. Demagnetization}

For the second part of the experiment, the clamp is attached to one phase shown in Fig. 3 and configured at the $100 \mathrm{mV} / \mathrm{A}$ range. Afterwards the circuit breaker is closed, allowing the circulation of currents in the armature winding. This point is shown in Fig. 8 at $55 \mathrm{~s}$ (a1) after the beginning of the recording period. A value of just around half an ampere is obtained. This means that the stator inductance $L_{\text {stator }}$ shown in Fig. 4 is very high and that its effect prevails in (1), that is, the machine is working in similar conditions as if it were a short circuit at its terminals. At $236 \mathrm{~s}$ the circuit breaker is opened again to check the emf waveform, with negligible changes observed, and closed soon thereafter (a2).

Fig. 8 b) focuses on the upper envelope of the current waveform to illustrate the downward trend of its amplitude, not appreciated in Fig. 8 a). The nitrogen level is maintained during this stage of the experiment by periodically replenishing the bucket and no other variations in the electrical circuit happen (the power dissipated in the resistors is below $0.2 \mathrm{~W}$ and should cause little heating). Therefore, one can conclude that the descending trend observed here corresponds to the reduction of the electromotive force as the stacks demagnetize due to the action of these same armature currents. This observed behaviour of surface mounted stacks, even at low armature currents, contributed to the selection of an interior mounted trapped-flux magnet rotor layout during the design phase of the first fully superconducting motor [6].

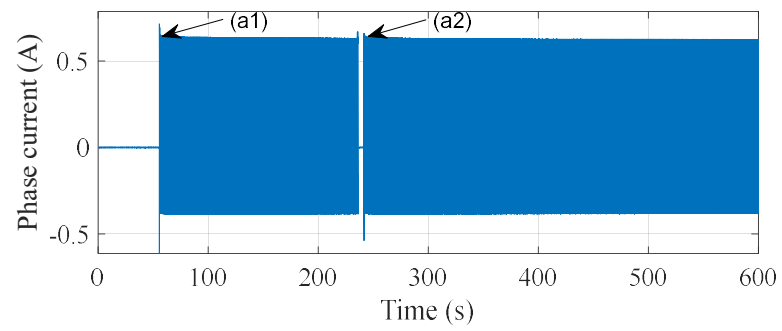

a)

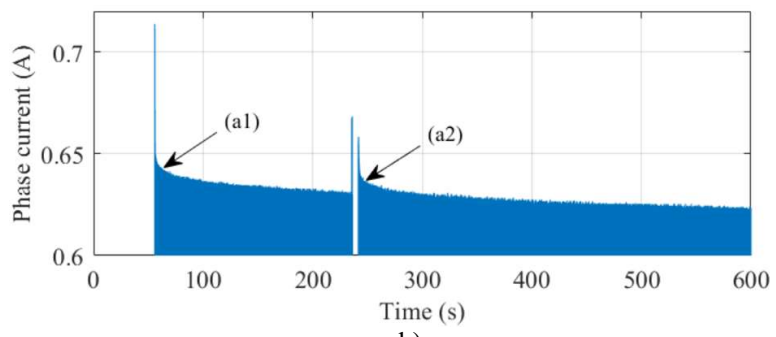

Fig. 8. Current waveform recorded during the experiment in one phase of the machine. During the first $50 \mathrm{~s}$ the emf is measured at open circuit. The load is connected in (a1) and reconnected in (a2) to allow circulating currents in the armature stator winding. Full waveform, a), upper envelope showing the descending trend of the stator currents' amplitude, b).

Table II compares the demagnetization rates obtained in this experiment with the ones carried out in [21]. Its third column shows the variation of the magnetic flux density tangential to the surface of the stacks, responsible for the demagnetization effect, computed by FE. The generation experiment carried out in this work shows higher values at the edges of the stacks, 45 $\mathrm{mT}$, since current circulates in all the coils. Nevertheless, the variation is lower, just $6 \mathrm{mT}$, in the central half of the superconducting region. Rotational frequency is also higher in this case, $16.33 \mathrm{~Hz}$ compared to $11 \mathrm{~Hz}$ in [21]. Furthermore, the fourth column states the observed descending rate, whereas the fifth shows the same values corrected. This is done by normalizing the electromotive force values from [21] in the proportion of the demagnetizing current ratio between both works, 0.6/3.5; whereas the current reduction measured in this 
work is divided by 4 in order to take into account that in [21] only one coil was used to measure the electromotive force, whereas here each phase has 4 of them connected in series. Finally, the last column presents the relative decrease in each magnitude, where it can be noticed that the demagnetization rate is half an order of magnitude lower if actual armature currents are used, compared to [21]. This is a logical outcome since in the experiment the main magnetic flux density component in the airgap is synchronous with the rotor movement. The rest of space harmonics: higher winding ones and ones caused by the reluctance variation in the airgap due to the slots, are the ones responsible for demagnetization $[3,15$, $16]$.

Lastly, the higher current peaks observed after each reconnection at $55 \mathrm{~s}$ and $241 \mathrm{~s}$, upon closer examination were identified as the effect of the asymmetrical components. They correspond to a DC offset, not a peak, and constitute the usual reaction of a generator to a sudden short circuit at its terminals. The short-circuited stator winding does not allow its flux linkage to change, producing DC components in each phase to preserve it. This DC component depends on the position of the rotor and hence is different for each reconnection. Furthermore, the rotor conductive areas now interact with this constant flux, so high currents are induced in these elements. Since the rotor is laminated, the only contributor must be the stacks themselves. The time constant obtained from the experimental data using a semilogarithmic plot is 0.375 seconds, more than 2 times larger than a permanent magnet machine, which reflects the strong interaction between the stacks and the stator winding under these short circuit conditions. This effect doesn't seem to permanently alter the state of the stacks.

\section{CONCLUSIONS}

Recently developed numerical approaches combining the $H$ and the $A$-formulations for simulating rotating electrical machines with superconductive regions in its rotor show a sufficient agreement with experimental results to validate its use in the initial design of these kind of devices. The differences observed are attributed to limitations on material data for the case reproduced and the severity of the pulse magnetization process available in the laboratory.

Furthermore, it has been experimentally demonstrated that the actual harmonics present in the airgap of a rotating electrical machine due to the stator's morphology cause demagnetization in rotor surface mounted stacks, even at low armature currents. The rate, however, is lower than in laboratory experiments using synthesized magnetic flux variations since in this case the main airgap magnetic flux density component is synchronous with the rotor movement.

Finally, the testing of machines with stacks as flux source under liquid nitrogen conditions is a straightforward procedure that may be easily improved with the experience gained in this work.

\section{REFERENCES}

[1] K. S. Haran et al., "High power density superconducting rotating machines-development status and technology roadmap," Supercond. Sci. Technol., 30123002 2017, (doi: 10.1088/1361-6668/aa833e).

[2] Song, X., Bührer, C. et al., "Designing and basic experimental validation of the world's first MW-class direct-drive superconducting wind turbine generator," IEEE Trans. Energy Convers., vol. 34, no. 4, pp. 2218-2225, 2019 (doi: 10.1109/TEC.2019.2927307)

[3] V. Climente-Alarcon, A. Patel, A. Baskys, B. A. Glowacki, "Design considerations for electric motors using stacks of high temperature superconducting tape as permanent magnets," IOP Conf. Ser.: Mater. Sci. Eng. 502 012182 2019. (doi:10.1088/1757-899X/502/1/012182).

[4] A. Patel, A. Baskys, T. Mitchell-Williams, A. McCaul, W. Coniglio, J. Hänisch, M. Lao, B. A. Glowacki, "A trapped field of $17.7 \mathrm{~T}$ in a stack of high temperature superconducting tape," Supercond. Sci. Technol, 31 92018 (doi: 10.1088/1361-6668/aad34c).

[5] Shengnan Zou, Magnetization of High Temperature Superconducting Trapped-Field Magnets, PhD Thesis, KIT Scientific Publishing, 2017. (Available on-line: https://publikationen.bibliothek.kit.edu/1000073152 )

[6] V. Climente-Alarcon, A. Smara, A. Patel, B. A. Glowacki, A. Baskys, T. Reis, "Field Cooling Magnetization and Losses of an Improved Architecture of Trapped-Field Superconducting Rotor for Aircraft Applications," AIAA Propulsion and Energy Forum and Exposition, Indianapolis, Indiana, August 2019, 2019-4313, (doi: 10.2514/6.20194313)

[7] A. Patel, V. Filar, I. Nizhankovskii, S. C. Hopkings, B. A. Glowacki, "Trapped fields greater than $7 \mathrm{~T}$ in a $12 \mathrm{~mm}$ square stack of commercial high-temperature superconducting tape", Appl.Phys.Letts., 102, 102601, 2013, (doi: 10.1063/1.4795016).

[8] A. Patel, A. Baskys, S. C. Hopkins, V. Kalitka, A. Molodyk, B. A. Glowacki, "Pulsed-Field Magnetization of Superconducting Tape Stacks for Motor Applications," IEEE Trans. Appl. Supercond., Vol. 25, no. 3 5203405, 2015 (doi: 10.1109/TASC.2015.2389142).

[9] A. Patel, A. Usoskin, A. Baskys, S. C. Hopkins, B. A. Glowacki, "Trapped field profiles for $40 \mathrm{~mm}$ wide superconducting tape pieces," $J$. Supercond. Nov. Magn., 28397-401, 2015.

[10] A. Perez, R. R. van der Woude, R. Dekker, "Rotor Cooling Concept for the ASuMED Superconductive Motor," IOP Conf. Ser.: Mater. Sci. Eng. Vol. 502, 012139, 2019, (doi: 10.1088/1757-899X/502/1/012139)

[11] R. Brambilla, F. Grilli, L. Martini, M. Bocchi, G. Angeli, "A finiteelement method framework for modelling rotating machines with superconducting windings," IEEE Trans. Appl. Supercond., Vol. 28, no. 5, 5207511, 2018, (doi: 10.1109/TASC.2018.2812884).

[12] V. Climente-Alarcon, A. Patel, A. Baskys, B. A. Glowacki, "Computation of Superconducting Stacks Magnetization in an Electrical Machine," IEEE Trans. Appl. Supercond., Vol. 29, no. 8, 5204506, Dec. 2019 (doi: 10.1109/TASC.2019.2923537).

[13] Y. Iwasa, "Case Studies in Superconducting Magnets, Design and Operational Issues," Second edition, Springer, 2009.

[14] A. Campbell, M. Baghdadi, A. Patel, D. Zhou, K. Y. Huang, Y. Shi, T. Coombs, "Demagnetisation by crossed fields in superconductors," Supercond. Sci. Technol., Vol. 30, no. 3, 034005, 2017.

[15] A. O. Di Tommaso, F. Genduso, R. Miceli, "A New Software Tool for Design, Optimization, and Complete Analysis of Rotating Electrical Machines Windings," IEEE Trans. Mag., Vol. 51, no. 4, 9401410, 2015.

[16] J. Pyrhönen, T. Jokinen, V. Hrabovcova, Design of Rotating Electrical Machines, Second Edition, New York, USA, Wiley, 2014

[17] M. Baghdadi, H. S. Ruiz, T. A. Coombs, "Crossed-magnetic field experiments on stacked second generation superconducting tapes: reduction of the demagnetization effects", Appl. Phys. Lett., Vol. 104, 232602, 2014, (doi: 10.1063/1.4879263).

[18] A. Baskys, A. Patel, B. A. Glowacki, "Measurements of crossed-field demagnetisation rate of trapped field magnets at high frequencies and below 77 K", Supercond. Sci. Technol., Vol. 31, 065011, 2018.

[19] M. Baghdadi, H. S. Ruiz, J. F. Fagnard, M. Zhang, W. Wang, T. A. Coombs, "Investigation of Demagnetization in HTS Stacked Tapes Implemented in Electric Machines as a Result of Crossed Magnetic Field," IEEE Trans. Appl. Supercond., Vol. 25, no. 3, 6602404, 2015.

[20] M. Baghdadi, H. S. Ruiz, T. A. Coombs, "Nature of the low magnetization decay on stacks of second generation superconducting tapes under crossed and rotating magnetic field experiments," Scientific Reports, Vol. 8, 1342, 2018, (doi: 10.1038/s41598-018-19681-8). 
[21] A. Smara, N. Mineev, V. Climente-Alarcon, A. Patel, A. Baskys, B. A. Glowacki, T. Reis, "Experimental Assessment of Rotor Superconducting Stack Demagnetization in LN2 Environment," Supercond. Sci. Technol., Vol. 32, no. 8, 08500, 2019, (doi: 10.1088/1361-6668/ab20bf).

[22] A. Arkkio, Analysis of Induction Motors Based on the Numerical Solution of the Magnetic Field and Circuit Equations, PhD. Thesis, 1986. (on-line: http://lib.tkk.fi/Diss/198X/isbn951226076X/).

[23] Z. Hong, A. M. Campbell, T. A. Coombs, Numerical solution of critical state in superconductivity by finite element software, Superconductor Science and Technology, Vol. 19, no. 12, 2006, pp. 1246-1252.

[24] J. H. Claassen, C. L. H. Thieme, "Magnetic properties of Ni-based substrates for HTS tape," Supercond. Sci. Technol., Vol. 21, 10500, 2008, (doi: 10.1088/0953-2048/21/10/105003)

[25] D. Wu, E. Chen, "Stator Design for a $1000 \mathrm{~kW}$ HTSC Motor With Airgap Winding," IEEE Trans. Appl. Supercond., Vol. 21, n 3. pp. 10931096, Jun. 2011, (doi: 10.1109/TASC.2010.2089962). 